\title{
Unusual synchronous skeletal muscle and lung metastasis in papillary thyroid cancer: A case report and review of the literature
}

\author{
JUN YANG $^{1}$, LIN-FA LI $^{1}$, XIU-MING ZHANG ${ }^{2}$, QIN XU $^{1}$, JUN ZHANG $^{1}$, \\ WAN-WEN WENG ${ }^{1}$ and MENG-JIE DONG ${ }^{3}$
}

\begin{abstract}
Departments of ${ }^{1}$ Nuclear Medicine, ${ }^{2}$ Pathology and ${ }^{3}$ Positron Emission Tomography Center, The First Affiliated Hospital, College of Medicine, Zhejiang University, Hangzhou, Zhejiang 310003, P.R. China
\end{abstract}

Received May 8, 2014; Accepted November 6, 2014

DOI: $10.3892 / \mathrm{ol} .2014 .2742$

\begin{abstract}
Papillary thyroid cancer (PTC) frequently metastasizes to the cervical lymph region and less often to the lung and bone. Metastasis to the skeletal muscles from PTC is extremely rare, especially concurrent lung and skeletal muscle metastases. The present study reports the case of a 31-year-old man with synchronous metastasis to the skeletal muscle and lung from PTC, six years following total thyroidectomy and consecutive ${ }^{131}$ Iodine treatments. Magnetic resonance imaging (MRI) revealed a $1.7 \times 1.2 \times 1.5 \mathrm{~cm}$ mass in the left gastrocnemius muscle, indicating a neurogenic tumor. The mass was subsequently resected and confirmed via histopathology to be metastatic PTC. We propose that, in the follow-up of patients with PTC, the measurable serum thyroglobulin level, whole body scan and other imaging modalities including MRI or positron emission tomography/computed tomography, must be closely monitored for potential distant metastases, particularly in cases of PTC with aggressive pathological behavior.
\end{abstract}

\section{Introduction}

The majority of thyroid cancers (90\%) are differentiated thyroid cancers (DTC), a term which includes both papillary and follicular cancer (1). Thyroid carcinoma accounts for $\sim 1 \%$ of all new malignant disease and $\sim 0.5$ and $1.5 \%$ of cancers in males and females, respectively (2). DTC progression is generally slow, with a low grade malignancy and excellent long-term survival rates. The overall 10-year survival rate of DTC is $>90 \%$ (3). Among DTC, the incidence of papillary thyroid cancer (PTC) was 7.9 per 100,000 individuals, the mortality rate was $\sim 0.4$ per 100,000 individuals and the overall

Correspondence to: Mr. Meng-jie Dong, Positron Emission Tomography Center, The First Affiliated Hospital, College of Medicine, Zhejiang University, 79 Qingchun Road, Hangzhou, Zhejiang 310003, P.R. China

E-mail: mengjiedong@126.com

Key words: papillary thyroid cancer, skeletal muscle metastasis, lung metastasis survival rate was $98.3 \%(4,5)$. Patients with DTC respond to total thyroidectomy, radioiodine ablation and levothyroxine suppression therapy. Distant metastases occur during follow-up in $2.2-23 \%$ of patients (6-8), and are usually identified in the lung and bone. Less frequently, they are detected in the brain, liver or other sites, and indicate a significantly poorer prognosis $(9,10)$. However, metastasis to the skeletal muscles from PTC is extremely rare, particularly synchronous lung and skeletal muscle metastases $(11,12)$. The present study reports the case of a 31-year-old male with PTC and concurrent metastasis to the left gastrocnemius muscle and lungs. Written informed consent was obtained from the patient.

\section{Case report}

In May 2007, a 31-year-old Chinese male presented to the Department of Surgical Oncology with palpable nodules of the thyroid in May 2007. An ultrasound (US) of the patient's neck revealed hypoechoic multinodules with microcalcification in the bilateral thyroid, the largest of which was located in the left lobe $(\sim 3.0 \mathrm{~cm})$. Cervical lymph node enlargement was also observed with microcalcification. Thyroid function tests indicated a euthyroid state. The patient had no family history of thyroid disease and denied any exposure to external or accidental radiation. Serum thyroglobulin $(\mathrm{Tg})$ level was measured at $82.2 \mathrm{ng} / \mathrm{ml}$ (normal range, $<55 \mathrm{ng} / \mathrm{ml}$ ), with negative anti-Tg antibodies ( $\mathrm{Tg} \mathrm{Ab}$ ). Following total thyroidectomy and central cervical node dissection, the patient was diagnosed with laryngotracheal invasion from PTC (pathological stage T4a N1a M1pul, 2010 AJCC) (13). The patient subsequently received radioiodine treatment four times (total dose, $24050 \mathrm{MBq}$ ). A whole body scan (WBS) revealed ${ }^{131}$ Iodine uptake in the lung (Fig. 1A), consistent with visible lesions on a computed tomography (CT) scan (Fig. 1B), which indicated pulmonary metastases.

The patient was referred to the First Affiliated Hospital (Hangzhou, China) in July 2013, with a three-month history of a slowly increasing mass in the left gastrocnemius muscle. On physical examination, the patient's vital signs were stable, and a soft mass of $\sim 1.5 \mathrm{~cm}$ in size was palpable in the left leg with no inflammatory surface. Serum Tg level was $80.2 \mathrm{ng} / \mathrm{ml}$ (normal range, $<1 \mathrm{ng} / \mathrm{ml}$ ). However, $\mathrm{TgAb}$ level was not available during this time. The results of tests for other biochemical tumor 
Table I. Synchronous lung and skeletal muscle metastases from PTC reported previously in the literature.

\begin{tabular}{lccccc}
\hline & Age, y/ & & $\begin{array}{c}\text { TNM } \\
\text { gender }\end{array}$ & Histology & $\begin{array}{c}\text { Survival after } \\
\text { after operation } \\
\text { diagnosis of } \\
\text { metastasis }\end{array}$ \\
\hline Bruglia et al $(11)$ & 44/male & PTC & T3N1bM0 & Yes & Biceps femoris, lung, skin, brain \\
Luo et al $(12)$ & 29/male & PTC & TXN1M1 & NA & Erector spinae, lung, kidney \\
Present case & $31 /$ male & PTC & T4N1aM1 & Yes & Gastrocnemius muscle, lung \\
\hline
\end{tabular}

NA, not available; PTC, papillary thyroid carcinoma; TX, Tumor size was not available; y, years.

A

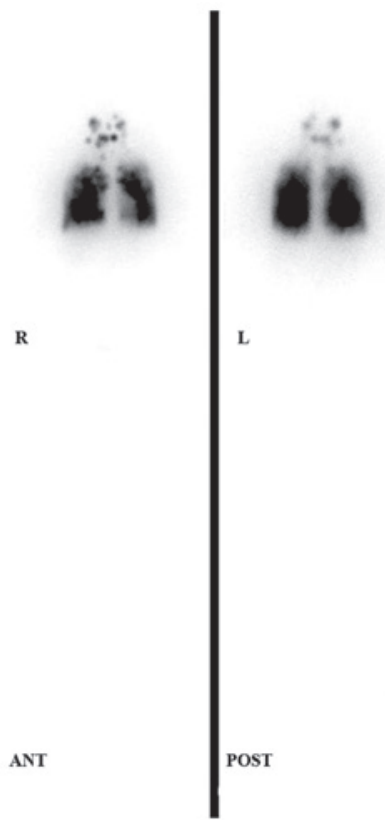

$\mathbf{B}$

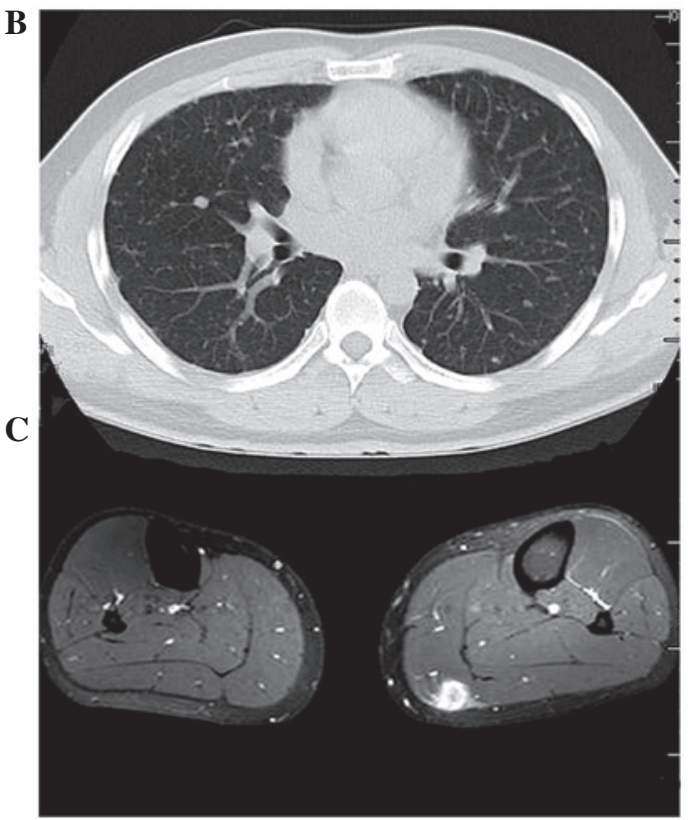

Figure 1. (A) ${ }^{131}$ Iodine whole-body scan, seven days after an oral therapeutic dose of $3700 \mathrm{MBq}{ }^{131}$ Iodine in 2007 , showed a diffuse uptake in the chest and focus of the neck. (B) Computed tomography scan of the thorax showed extensive metastatic disease of the lungs. (C) Magnetic resonance imaging showed gastrocnemius muscle metastasis in the left leg.
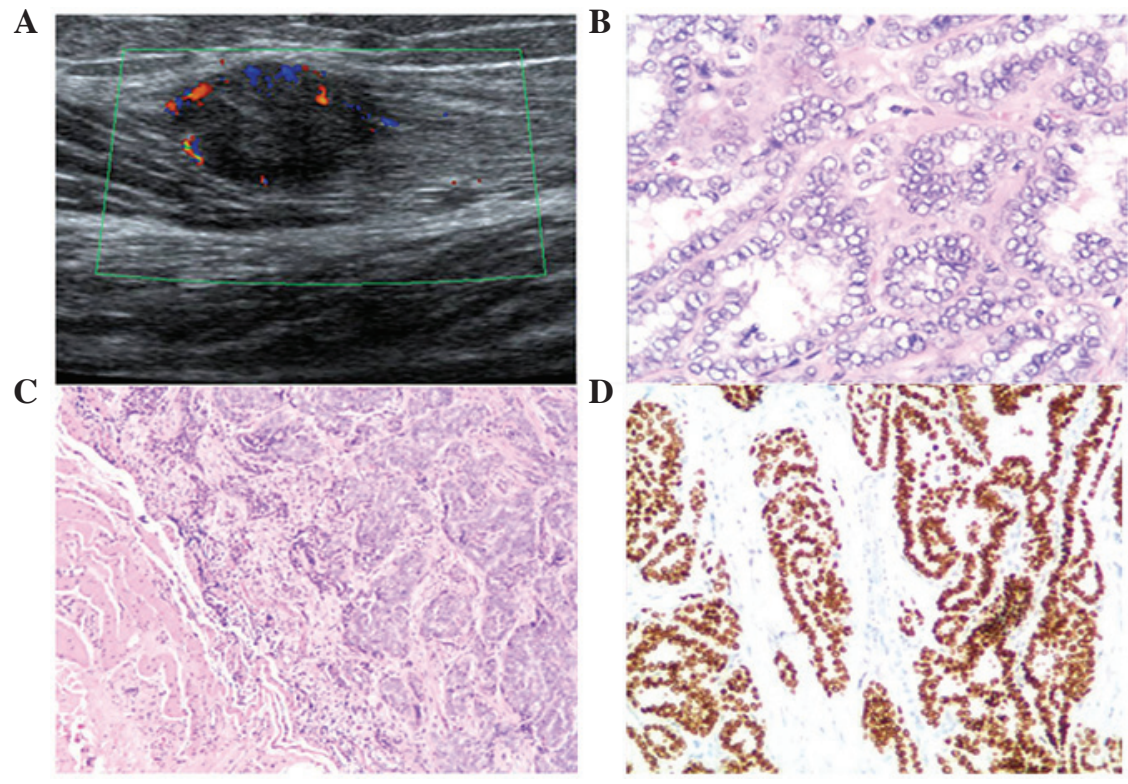

Figure 2. (A) Doppler ultrasound revealed a hypoechoic lesion with peripheral hypervascularity. (B) Infiltrating clusters of papillary tumor cells in the skeletal muscle (HE staining; magnification, x50). (C) Typical nuclear inclusions and grooves (HE staining; magnification, x200). (D) Immunohistochemistry showed tumor cells positive for thyroid transcription factor 1 . HE, hematoxylin and eosin. 
markers, including $\alpha$-fetoprotein $(2.8 \mathrm{ng} / \mathrm{ml}$; normal range, $<20.0 \mathrm{ng} / \mathrm{ml})$, carcino-embryonic antigen $(1.9 \mathrm{ng} / \mathrm{ml}$; normal range, $<5.0 \mathrm{ng} / \mathrm{ml})$, sugar antigen 199 (4.2 U/ml; normal range, $<37 \mathrm{U} / \mathrm{ml}$ ), sugar antigen 125 (13.0 U/ml; normal range, <35 U/ $\mathrm{ml})$ and total prostate specific antigen $(2.103 \mathrm{ng} / \mathrm{ml}$; normal range, $<4.0 \mathrm{ng} / \mathrm{ml}$ ), were within normal limits. Magnetic resonance imaging (MRI) revealed a soft-tissue mass that was markedly low in signal intensity on T1-weighted images, and enhanced after administration of contrast material (Fig. 1C). Doppler US showed a 1.7x1.2x1.5 cm solitary, hypoechoic nodule with peripheral hypervascularity (Fig. 2A). A diagnosis of neurogenic tumor was highly suspected due to the morphological features of the mass. A local marginal resection was subsequently performed. Histopathology revealed the mass to be a metastatic papillary tumor (Fig. 2B and C), and immunohistochemical examination showed that the cells were positive for thyroid transcription factor-1 (TTF-1) (Fig. 2D), indicating gastrocnemius muscle metastasis from PTC. Concurrently, CT imaging of the chest revealed innumerable, moderately well-circumscribed nodules in the lung with high ${ }^{131}$ Iodine uptake, indicating tumor metastases. After surgery, the patient was treated with ${ }^{131}$ Iodine $(6660 \mathrm{MBq})$. The patient's Tg levels decreased to $40.6 \mathrm{ng} / \mathrm{ml}$ (normal range, $<1 \mathrm{ng} / \mathrm{ml}$ ) and pulmonary metastases were stable after six months of follow-up.

\section{Discussion}

Papillary thyroid cancer is commonly associated with lymphatic spread to regional lymph nodes. Distant metastases occur more rarely, usually involving the lungs and bone (1-7\% of patients) (14), but occasionally occurs in the brain, sphenoid sinus, orbit, adrenal, kidney and ovary (15-18). A retrospective review of the literature revealed only two case reports of muscle metastases arising from PTC $(11,12)$. It has been hypothesized that skeletal muscle is a hostile environment for proliferating cancer cells, due to muscle motion and unadapted muscle $\mathrm{pH}$ (19), which may explain the lack of such cases. Synchronous lung and skeletal muscle disease from PTC is an extremely rare manifestation (18). To the best of our knowledge, only two cases have been previously reported (Table I). Bruglia et al (11) reported the case of a 44-year-old male with PTC, with a poorly differentiated thyroid carcinoma component and metastases to the thigh muscle, skin, lung, mediastinum and brain. The aggressiveness of the tumor led to mortality eight years following total thyroidectomy. Luo et al (12) reported the case of a 29-year-old male patient who had unusual metastasis sites in the lung, kidney and erector spinae from PTC. The authors concluded that suspected tumors must be considered as potential metastases from thyroid carcinomas in the clinical setting. The current study reports a rare case of PTC associated with diffuse metastases to the lung and gastrocnemius muscle, six years following total thyroidectomy and radioiodine treatment.

The particularly good prognosis and long-term survival of PTC patients are significantly reduced in those with distant metastasis (DM). The early differential diagnosis of distant metastases of thyroid carcinoma remains difficult using common diagnostic modalities such as US, Tg levels and ${ }^{131} \mathrm{I}-\mathrm{WBS}$. Although postoperative serum Tg levels and
${ }^{131}$ I-WBS scans are sensitive methods in the detection of metastatic disease, accurately localizing the source of the abnormalities can be problematic, particularly in soft tissues. In addition, radioiodine-negative thyroid cancer may account for $\leq 20 \%$ of cases of DTC, which presents challenges in the localization of metastatic lesions, even if the serum $\mathrm{Tg}$ level is evaluated (20). Other imaging modalities, including MRI and 2-deoxy-2-( $\left({ }^{18} \mathrm{~F}\right)$ fluoro-D-glucose $\left({ }^{18} \mathrm{FDG}\right)$-positron emission tomography (PET), may also be valuable in the follow-up of thyroid cancer metastases, particularly in patients with elevated Tg levels and normal radioiodine WBS (21).

Various factors may contribute to the development of DM, including large and multifocal primary tumors, extrathyroidal extension, aggressive histology and advanced age (>45 years) (22). Due to the rarity of synchronous lung and skeletal muscle metastasis of PTC, characteristic risk factors have not yet been identified. In the present case, postoperative pathology revealed that DTC had invaded the laryngotracheal region. Microscopic or gross invasion of the tumor into the perithyroid, aggressive histology or vascular invasion may be associated with an intermediate-high risk of recurrence (23). Mortality is increased in patients with distant metastases, particularly in those aged $>45$ years (6). Notably, the patient in the present study was only 37 years of age, and remains alive six years following total thyroidectomy and radioiodine treatment, which may support this observation.

In conclusion, this study presents a rare case of synchronous skeletal muscle and lung metastasis from PTC. We propose that Tg measurement, WBS and other imaging modalities, including US, MRI and ${ }^{18}$ FDG-PET, must be utilized during follow-up of patients with PTC, to detect possible uncommon distant metastases, particularly in cases of PTC that exhibit aggressive pathological behavior.

\section{References}

1. Sherman SI: Thyroid carcinoma. Lancet 361: 501-511, 2003.

2. Figge J: Epidemiology of thyroid cancer. In: Thyroid Cancer: A Comprehensive Guide to Clinical Management. Wartofsky L (ed). Humana Press, Totowa, NJ, pp77-83, 1999.

3. Gilliland FD, Hunt WC, Morris DM and Key CR: Prognostic factors for thyroid carcinoma: A population-based study of 15,698 cases from the Surveillance, Epidemiology and End Results (SEER) program 1973-1991. Cancer 79: 564-573, 1997.

4. Chen AY, Jemal A and Ward EM: Increasing incidence of differentiated thyroid cancer in the United States, 1988-2005. Cancer 115: 3801-3807, 2009.

5. Albores-Saavedra J, Henson DE, Glazer E and Schwartz AM: Changing patterns in the incidence and survival of thyroid cancer with follicular phenotype - papillary, follicular, and anaplastic: a morphological and epidemiological study. Endocr Pathol 18: 1-7, 2007.

6. Nixon IJ, Whitcher MM, Palmer FL, et al: The impact of distant metastases at presentation on prognosis in patients with differentiated carcinoma of the thyroid gland. Thyroid 22: 884-889, 2012.

7. Mihailovic J, Stefanovic L and Malesevic M: Differentiated thyroid carcinoma with distant metastases: probability of survival and its predicting factors. Cancer Biother Radiopharm 22: 250-255, 2007.

8. Goffredo P, Sosa JA and Roman SA: Differentiated thyroid cancer presenting with distant metastases: a population analysis over two decades. World J Surg 37: 1599-1605, 2013.

9. Lin JD, Chao TC, Chou SC and Hsueh C: Papillary thyroid carcinomas with lung metastases. Thyroid 14: 1091-1096, 2004.

10. Pittas AG, Adler M, Fazzari M, Tickoo S, Rosai J, Larson SM and Robbins RJ: Bone metastases from thyroid carcinoma: clinical characteristics and prognostic variables in one hundred forty-six patients. Thyroid 10: 261-268, 2000. 
11. Bruglia M, Palmonella G, Silvetti F, et al: Skin and thigh muscle metastasis from papillary thyroid cancer. Singapore Med J 50: e61-e64, 2009.

12. Luo Q, Luo QY, Sheng SW, et al: Localization of concomitant metastases to kidney and erector spinae from papillary thyroid carcinoma using (131)I-SPECT and CT. Thyroid 18: 663-664, 2008.

13. NCCN guidelines: Thyroid carcinoma. Version 3, 2011.

14. Tahmasebi FC, Farmer P, Powell SZ, et al: Brain metastases from papillary thyroid carcinomas. Virchows Arch 462: 473-480, 2013.

15. Liou MJ,Lin JD, Chung MH,Liau CT and Hsueh C: Renal metastasis from papillary thyroid microcarcinoma. Acta Otolaryngol 125: 438-442, 2005 .

16. Brogioni S, Viacava P, Tomisti L, et al: A special case of bilateral ovarian metastases in a woman with papillary carcinoma of the thyroid. Exp Clin Endocrinol Diabetes 115: 397-400, 2007.

17. Rocha Filho FD, Lima GG, Ferreira FV, Lima MG and Hissa MN Orbital metastasis as primary clinical manifestation of thyroid carcinoma - case report and literature review. Arq Bras Endocrinol Metabol 52: 1497-1500, 2008.

18. Argibay Vázquez S, Lancha Hernández C and Martínez Muñiz A Metastases in the sphenoidal sinus in a patient with papillary thyroid cancer. Clin Transl Oncol 7: 324-327, 2005.
19. Seely S: Possible reasons for high resistance of muscle to cancer. Med Hypotheses 6: 133-137, 1980.

20. Bae SY, Lee SK, Koo MY, et al: Distant, solitary skeletal muscle metastasis in recurrent papillary thyroid carcinoma. Thyroid 21: 1027-1031, 2011.

21. Dong MJ,LiuZF,Zhao K, et al: Value of 18F-FDG-PET/PET-CT in differentiated thyroid carcinoma with radioiodine-negative whole-body scan: a meta-analysis. Nucl Med Commun 30: 639-650, 2009

22. Toubert ME, Hindie E, Rampin L, Al-Nahhas A and Rubello D Distant metastases of differentiated thyroid cancer: diagnosis, treatment and outcome. Nucl Med Rev Cent East Eur 10: 106-109, 2007.

23. American Thyroid Association (ATA) Guidelines Taskforce on Thyroid Nodules and Differentiated Thyroid Cancer. Cooper DS, Doherty GM, Haugen BR, et al: Revised American Thyroid Association management guidelines for patients with thyroid nodules and differentiated thyroid cancer. Thyroid 19: 1167-1214, 2009. 